Final Report LDRD 02-ERD-013 Dense Plasma Characterization by X-ray Thomson Scattering

O.L. Landen, S.H. Glenzer, G. Gregori, S.M. Pollaine, J.H. Hammer, F. Rogers, N.B. Meezan, H.-K. Chung, R.W. Lee

February 18, 2005 
This document was prepared as an account of work sponsored by an agency of the United States Government. Neither the United States Government nor the University of California nor any of their employees, makes any warranty, express or implied, or assumes any legal liability or responsibility for the accuracy, completeness, or usefulness of any information, apparatus, product, or process disclosed, or represents that its use would not infringe privately owned rights. Reference herein to any specific commercial product, process, or service by trade name, trademark, manufacturer, or otherwise, does not necessarily constitute or imply its endorsement, recommendation, or favoring by the United States Government or the University of California. The views and opinions of authors expressed herein do not necessarily state or reflect those of the United States Government or the University of California, and shall not be used for advertising or product endorsement purposes.

This work was performed under the auspices of the U.S. Department of Energy by University of California, Lawrence Livermore National Laboratory under Contract W-7405-Eng-48. 


\title{
Final Report LDRD 02-ERD-013
}

\section{Dense Plasma Characterization by X-ray Thomson Scattering*}

\author{
O.L. Landen, S.H. Glenzer, G. Gregori, S.M. Pollaine, J.H. Hammer, F. Rogers, \\ N.B. Meezan, H.-K. Chung and R.W. Lee \\ Lawrence Livermore National Laboratory, Livermore, CA USA 94551-0808
}

\begin{abstract}
We have successfully demonstrated spectrally-resolved x-ray scattering in a variety of dense plasmas as a powerful new technique for providing microscopic dense plasma parameters unattainable by other means. The results have also been used to distinguish between ionization balance models. This has led to 10 published or to be published papers, 8 invited talks and significant interest from both internal and external experimental plasma physicists and the international statistical plasma physics theory community.
\end{abstract}

\footnotetext{
*This work was performed under the auspices of U.S. Department of Energy by the University of California Lawrence Livermore National Laboratory under Contract No. W-7405-Eng-48.
} 


\section{Introduction}

The full range of plasmas, from Fermi degenerate, to strongly coupled, to high temperature ideal gas plasmas are present at high density in a variety of laboratory [1-7] and astrophysical environments [8-10]. The Fermi degenerate plasmas can be characterized by $\mathrm{T}_{\mathrm{e}}<\mathrm{T}_{\mathrm{F}}$, the strongly coupled plasmas by a combination of $T_{e}>T_{F}$ and the ratio $\square_{e e}$ of the Coulomb potential energy between free electrons to the kinetic energy of the free electrons being $>1$, and the ideal plasmas by $\square_{e x}<1$. These are states of matter occurring at some location during the interaction between intense lasers and a solid and are therefore of general interest for any research program that uses intense lasers. These are also regimes accessed by the DT fuel during an ICF implosion.

Various statistical mechanics models [11-13] differ by factors of several in the predicted electron-ion collisionality in this regime. Material properties such as electrical [14-17] and thermal conductivity [18,19], opacity [20-23], and equationof-state (EOS) $[24,25]$ have been studied in this regime to attempt to resolve theoretical and calculational uncertainties. However, the usefulness of such

measurements has been impaired because of the lack of an independent measurement of temperature and density. For example, surface probing of any overdense plasmas is difficult to interpret because density gradient scalelengths of the order of $\square / 2 \square$ dramatically modify observables such as reflectivity and phase modulation. Internal x-ray probing for plasmas at densities near solid and above has relied, so far, on continuum edge spectroscopy and extended x-ray absorption fine-structure (EXAFS), line shape spectroscopy, or non-spectrally resolved x-ray scattering [26-29]. However, the interpretation of results from all 
such techniques relies on knowledge of the ionization balance, density and temperature.

By extending the power of spectrally resolved Thomson scattering to the $\mathrm{x}$ ray regime, we have shown by calculating scattering spectra in the random phase approximation that a direct measurement of the ionization state and the temperature of dense plasmas can be achieved. Specifically, by spectrally discriminating between the coherent and Compton-downshifted [30-34] and Doppler-broadened Thomson scattered components, we can gather information on many microscopic parameters, including the free and bound electron densities and fractions, temperature, plasma flow velocity, and plasma collisionality. These direct measurements of microscopic parameters of dense plasmas could eventually be used to properly interpret laboratory measurements of material properties such as thermal and electrical conductivity, EOS and opacity.

\section{X-ray Thomson/Compton Scattering}

Incoherent Thomson scattering [35-38] at a probe wavelength $\square$ and angle $\square$ is characterized by the scattering parameter $\square=\square_{\mathrm{s}} / 2 \square \square_{\mathrm{D}}<1$, where $\square_{\mathrm{s}}=$ $\square / 2 \sin (\square / 2)$ is the scattering correlation length and $\square_{D}$ is the plasma Debye

correlation length. By switching from current UV lasers near $2400 \AA$ to an x-ray probe at $2.4 \AA$, we can now probe solid density plasmas with Debye lengths of the order of the interparticle spacing or shorter $(1 \AA)$. In Figure 1, the large plasma parameter space available to X-ray scattering at a probe wavelength of $2.4 \AA$ is shown. The gray region denotes the parameter space for the ICF fuel 
during compression, reaching super-solid densities. The black regions denote the parameter space for plasmas probed as part of this LDRD research. All of these are in the scattering regime $\square<1$, for which spectrally-resolved incoherent Thomson scattering provides direct information on the electron velocity distribution function $\mathrm{f}(\mathrm{v})$ of free electrons from the Doppler shifts experienced by scattered probe photons. In particular the width and form of the velocity distribution will in general be a function of both $\mathrm{T}_{\mathrm{e}}$ and the Fermi energy $\mathrm{T}_{\mathrm{F}}$, hence also a function of $\mathrm{n}_{\mathrm{e}} \sim \mathrm{T}_{\mathrm{F}}^{3 / 2}$.

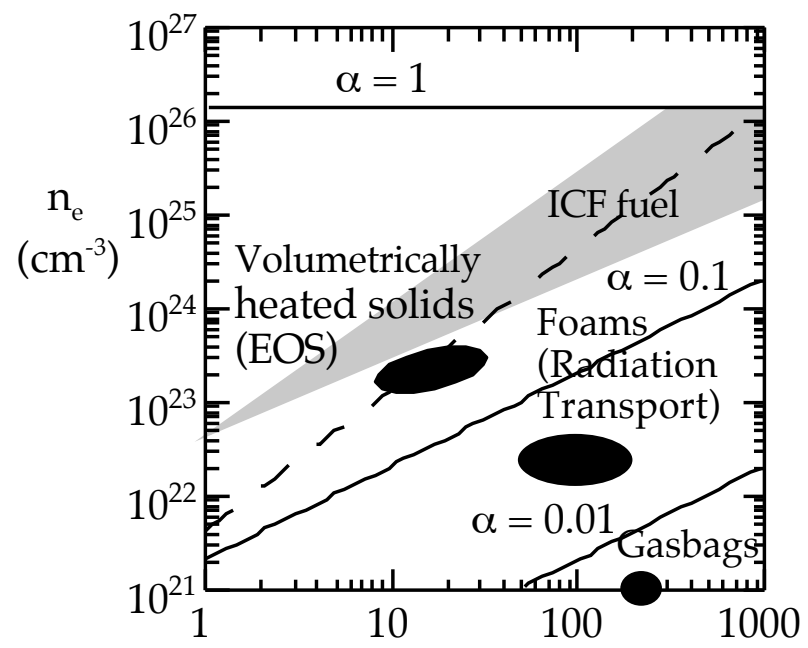

Figure 1: Electron density and temperature parameter space showing plasma regimes accessible. Dashed line denotes $\mathrm{T}_{\mathrm{e}}$ $=\mathrm{T}_{\mathrm{F}}$ boundary. Solid lines give scattering parameter for $\square=180^{\circ}$ Thomson scattering for $\square=2.4 \AA$ probe. Black regions denote plasmas already probed. Gray region denotes ICF fuel regime to be explored in future.

\section{Results and Summary}

Over the three year LDRD grant, we have developed at the Omega facility various isochorically heated uniform high density low $\mathrm{Z}$ plasmas that are probed by closely-coupled x-ray line radiation sources [IV, VI], for which the near backscattered x-rays are resolved by high efficiency spectrometers [IX]. In parallel, we have developed an integrated theoretical framework for analyzing the scattered spectra for extracting ionization state and electron temperature [I], [II], [III]. The results have been used to distinguish between ionization balance 
models [VI], [VII], [VIII] for Be and C, and the ionization state and temperature of radiatively heated $\mathrm{C}$ foams and $\mathrm{CH}$ gasbags $[\mathrm{X}]$. The success of this project has led to a further funded LDRD on using forward collective scattering $(\square>1)$ to probe solid density plasmons, and to proposals for scattering using future free-electron x-ray lasers [V]. Other groups around the world are now beginning to apply this technique to measure the adiabat of ICF ablators and eventually to measure the ionization balance of shocked $D_{2}$, an important outstanding experimental issue. Finally, we have designed experiments for applying x-ray scattering to measuring the adiabat of imploded DT ICF fuel at NIF that can reach 100x solid densities. These latter experiments would use a PW beam on NIF to create $50 \mathrm{keV} \mathrm{K} \square$ x-rays for probing hot imploded cores. 


\section{Publications resulting from this LDRD:}

I) G. Gregori, S.H. Glenzer, et. al., "Calculations and Measurements of X-Ray Thomson Scattering Spectra in Warm Dense Matter", in Proceedings of $16^{\text {th }}$ International Conference on Spectral Lineshapes, C.A. Back Ed., (AIP Press, New York, 2002) pp. 359-368. UCRL-JC-148076

II) G. Gregori, S.H. Glenzer, W. Rozmus, R.W. Lee, and O.L. Landen, “Theoretical Modelling of X-Ray Scattering as a Dense Matter Probe", Phys. Rev. E 67 (2003) 026412. UCRL-JC-147395

III) G. Gregori, R.W. Lee, S.H. Glenzer, and O.L. Landen, “Strong Coupling Corrections in the Analysis of X-Ray Scattering Measurements", J. Phys. A 36 (2003) 5971. UCRL-JC-150760

IV) S.H. Glenzer, G. Gregori, R.W. Lee, F.J. Rogers, et. al., "Demonstration of Spectrally Resolved X-Ray Scattering in Dense Plasmas", Phys. Rev. Lett. 90 (2003) 175002. UCRL-JC-149694-Rev1

V) R.W. Lee, S.J. Moon, H.K. Chung, W. Rozmus, H.A. Baldis, G. Gregori, R.C. Cauble, O.L. Landen, J.S. Wark, A. Ng, S.J. Rose, C. L. Lewis, D. Riley, J.-C. Gauthier, P. Audebert, "Finite Temperature Dense Matter Studies on Next Generation Light Sources," Opt. Soc. Amer. 20 (2003) 770. UCRL-JC-147883-Rev1 
VI) S.H. Glenzer, G. Gregori, F.J. Rogers, D.H. Froula, S.M. Pollaine, R.S. Wallace, and O.L. Landen, "X-Ray Thomson Scattering from Solid Density Plasmas", Phys. Plasmas 10 (2003) 2433. UCRL-JC-151045

VII) G. Gregori, S.H. Glenzer, F.J. Rogers, S.M. Pollaine, O.L. Landen, C. Blancard, G. Faussurier, P. Renaudin, S. Kuhlbrodt, and R. Redmer, "Electronic Structure Measurements of Dense Plasmas", Phys. Plasmas 11 (2004) 2754. UCRL-CONF-155622

VIII) G. Gregori, S.H. Glenzer, F.J. Rogers, O.L. Landen, C. Blancard, G. Faussurier, P. Renaudin, S. Kuhlbrodt, and R. Redmer, "Electronic Structure Measurement of Solid Density Plasmas using X-Ray Scattering", in Proceedings of Inertial Fusion Sciences and Applications 2003, B.A. Hammel Ed., (American Nuclear Society, Illinois, 2004) pp. 902-906. UCRL-JC-152388

IX) A. Pak, G. Gregori, J. Knight, K. Campbell, D. Price, B. Hammel, O.L. Landen, and S.H. Glenzer, "X-ray Line Measurements with High Efficiency Bragg Crystals", Rev. Sci. Instrum. 75 (2004) 3747. UCRL-CONF-203748

X) G. Gregori, S.H. Glenzer, H.-K. Chung, R.W. Lee, N.B. Meezan and O.L. Landen, "Measurement of the Ionization Balance in High Temperature Plasma Mixtures by Temporally Resolved Scattering", to be published in J. Quantit. Spectrosc Radiat Trans (2005). UCRL-JRNL-208894 


\section{References}

1) Setsuhara $Y$, Azechi H, Miyanaga N et al. Laser Part. Beams 1990:8:609.

2) Lindl J. Phys. Plasmas 1995;2:3933.

3) Peyrusse O, Busquet M, Kieffer JC et al. Phys. Rev. Lett. 1995;75:3862.

4) Woolsey NC, Asfaw A, Hammel BA et al. Phys. Rev. E 1996;53:6396.

5) Meyer-ter-Vehn J, Oparin A, and Aoki T. in 12th International Conference on Laser Interaction and Related Plasma Phenomena, vol. 369. Woodbury NY: AIP, 1996. p. 347.

6) Nantel M, Ma G, Gu S et al. Phys. Rev. Lett. 1998;80:4442.

7) Theobald W, Hassner R, Kingham R et al. Phys. Rev. E 1999;59:3544.

8) Ichimaru. S. Rev. Mod. Phys 1982;54:1017.

9) van Horn HM. The Equation of State in Astrophysics. Cambridge:Cambridge University Press, $1994 .$.

10) Rose SJ. Astrophys. J. Lett. 1995;453:L45.

11) Linnebur EJ and Duderstadt JJ. Phys. Fluids 1973;16:665.

12) Theimer O and Behl YK. Plasma Phys. 1977;19:1119.

13) Cauble R and Boercker DB. Phys. Rev. A 1983;28:944.

14) DeSilva AW and Kunze HJ. Phys. Rev. E 1994;49:4448.

15) Krisch I and Kunze HJ. Phys. Rev. E 1998;58:6557.

16) DeSilva AW and Katsouros JD.Phys. Rev. E 1998;57:5945.

17) Benage JF, Jr., Shanahan WR, Sherwood EG et al. Phys. Rev. E 1994;49:4391.

18) Rozmus W and Offenberger AA. Phys. Rev. A 1985;31:1177.

19) Ng A, Forsman A, and Chiu G. Phys. Rev. Lett. 1998;81:2914.

20) Mostovych AN, Kearney KJ, Stamper JA et al. Phys. Rev. Lett. 1991;66:612.

21) Eidmann K, Bar-Shalom A, Saemann A et al. Europhys. Lett. 1998;44:459. 
22) Gauthier P, Rose SJ, Sauvan P et al. Phys. Rev. E 1998;58:942.

23) Davidson SJ, Nazir K, Rose SJ et al. J. Quant. Spectrosc. Radiat. Trans. 2000;65:151.

24) Liberman D and Albritton J. J. Quant. Spectrosc. Radiat. Trans. 1994;51:197.

25) Perrot F and Dharma-Wardana MWC. Phys. Rev. E 1995;52:5352.

26) Nardi E. Phys. Rev. A 1991;43:1977.

27) Riley D, Woolsey NC, McSherry D, Weaver I, Djaoui A, and Nardi E. Phys. Rev. Lett.. 2000;84:1704.

28) Nardi E, Zinamon Z, Riley D et al. Phys. Rev. E 1998;57:4693.

29) Riley D, Woolsey NC, McSherry D et al. J. Quant. Spectrosc. Radiat. Trans. 2000;65:463.

30) Bloch F. Phys. Rev. 1934;46:674.

31) Weigele WJ, Tracy PT, and Henry EM. Am. J. Phys. 1966;34:1116.

32) Chandrasekhar S. Proc. Roy. Soc. 1929;A125:231.

33) Issolah A, Levy B, Beswick A, and Loupias G, Phys. Rev. A 1988;38:4509.

34) Landen Ol, Glenzer SH, Edwards MJ, Lee RW et al. J. Quant. Spectrosc. Radiat. Trans. 2001;71:465.

35) Evans DE and Katzenstein J. Rep. Prog. Phys. 1969;32:207.

36) Cameron SM, Tracy MD, Estabrook KG et al. Rev. Sci. Instrum. 1992;63:5259.

37) Glenzer SH., Rozmus W, MacGowan BJ et al. Phys. Rev. Lett. 1999;82:97.

38) Montgomery DS, Johnson RP, Cobble JA et al. Laser Part. Beams 1999;17:349. 